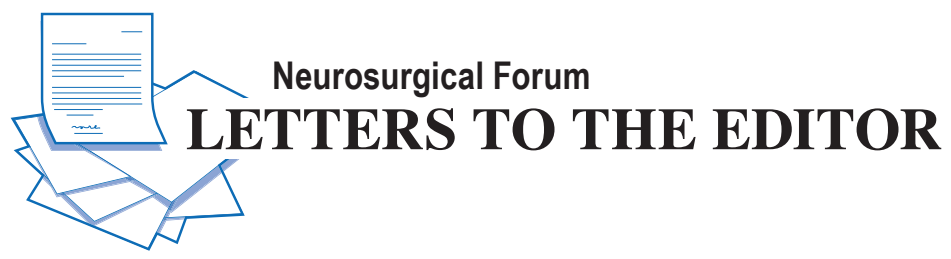

\section{Graft selection in cerebral revascularization surgery}

TO THE EDITOR: We read with great interest the recent article by Stapleton et al. ${ }^{1}$ in which a comparative study of auto- and allo-saphenous vein grafts was conducted with a short-term follow-up (Stapleton CJ, Hussein AE, Behbahani M, et al. Comparative efficacy of autologous versus cadaveric saphenous vein grafts in cerebral revascularization surgery. J Neurosurg. Published online May 22, 2020. doi:10.3171/2020.3.JNS192546). The investigators disclosed that the autologous vein graft (AVG) achieved more robust blood flow, but a higher patency rate was noted in the xenogeneic vein graft (XVG) within 3 months postoperatively. They also concluded that allografts are a viable candidate when autografts are unavailable. We commend the authors for such a novel clinical study in terms of bypass graft selection, but some issues of this research should be addressed.

Cadaveric or artificial vessel grafts have been widely used in peripheral and cardiac surgery, as the researchers described. The benefits of applying xenogeneic grafts in bypass surgery include the avoidance of a second incision and, more importantly, the precise selection of grafts with a desired length and diameter. As is well known, the US is one of only a few countries with sufficient resources to have the capability to obtain a large number of XVGs to apply in clinical practice, which means that the fact that the rest of the countries in the world have limited resources in terms of human body donations significantly impedes the utilization of these grafts. In addition, ethical issues are sometimes a concern due to the strict religious backgrounds in some regions.

The overall patency rate of this series was less than $75 \%$ during only 2.2 months of follow-up, which was significantly lower than that of arterial grafts after long-term observation ( $>3$ years). ${ }^{2}$ This problem can be magnified within flow replacement bypass for complex aneurysms or tumors because the main arterial trunk has been sacrificed; furthermore, if the graft is occluded, this results in a high risk of ischemia in brain tissue. In addition, we may not strongly agree with the investigators in terms of their preferred application of venous grafts due to the availability of multiple arterial grafts, ${ }^{3}$ including grafts of the radial artery, ${ }^{4-7}$ the descending branch of the lateral femoral circumflex artery, ${ }^{8}$ and the tibial artery, ${ }^{9,10}$ which have been attempted with better outcomes than those of venous grafts. Moreover, although more than $25 \%$ of patients experienced graft occlusion in this series, their clinical outcomes or complications were not described or analyzed. Last, the investigators described the internal maxillary artery (IMaxA) as an interposition graft and simultaneously cited our previous publication. ${ }^{5}$ From our perspective, the IMaxA has been one of the most popular donors other than the grafts used in cases of high-flow bypass. ${ }^{2-7}$

In conclusion, we do not discredit allograft utilization but hope that cadaveric arterial grafts such as grafts of the radial artery or superficial temporal artery trunk can be implemented in the near future.

Long Wang, MD, PhD

SanBo Brain Hospital, Capital Medical University, Beijing, China

Lujun Jing, MD

Beijing Electric Power Hospital, Capital Medical University, Beijing, China

Huaiyu Sun, MD

TieMei General Hospital of Liaoning Health Industry Group, Liaoning, China

Xiang'en Shi, MD, PhD

SanBo Brain Hospital, Capital Medical University, Beijing, China

\section{References}

1. Stapleton CJ, Hussein AE, Behbahani M, et al. Comparative efficacy of autologous versus cadaveric saphenous vein grafts in cerebral revascularization surgery. J Neurosurg. Published online May 22, 2020. doi:10.3171/2020.3.JNS192546

2. Wang L, Lu S, Qian H, et al. Internal maxillary artery bypass with radial artery graft treatment of giant intracranial aneurysms. World Neurosurg. 2017;105:568-584.

3. Wang L, Shi X, Qian H. Graft selection in high-flow internal maxillary artery bypass. Letter. Oper Neurosurg (Hagerstown). 2018;14(1):10-11.

4. Wang L, Shi X, Qian H. Flow reversal bypass surgery: a treatment option for giant serpentine and dolichoectatic aneurysms-internal maxillary artery bypass with an interposed radial artery graft followed by parent artery occlusion. Neurosurg Rev. 2017;40(2):319-328.

5. Wang L, Cai L, Lu S, et al. The history and evolution of internal maxillary artery bypass. World Neurosurg. 2018;113:320-332.

6. Wang L, Qian H, Shi X. The "SHI" internal maxillary 
bypass for giant fusiform middle cerebral artery bifurcation aneurysms: 2-dimensional operative video. World Neurosurg. 2019;122:58.

7. Wang L, Lu S, Cai L, et al. Internal maxillary artery bypass for the treatment of complex middle cerebral artery aneurysms. Neurosurg Focus. 2019;46(2):E10.

8. Ravina K, Kramer DR, Strickland BA, et al. Complex revascularization for idiopathic intracranial occlusive disease with unruptured, fusiform anterior cerebral artery and middle cerebral artery aneurysms: 3-dimensional operative video. World Neurosurg. 2019;126:496.

9. Ramanathan D, Starnes B, Hatsukami T, et al. Tibial artery autografts: alternative conduits for high flow cerebral revascularizations. World Neurosurg. 2013;80(3-4):322-327.

10. Strickland BA, Bakhsheshian J, Rennert RC, et al. Descending branch of the lateral circumflex femoral artery graft for posterior inferior cerebellar artery revascularization. Oper Neurosurg. 2018;15(3):285-291.

\section{Disclosures}

The authors report no conflict of interest.

\section{Correspondence}

Xiang'en Shi: shixen@ccmu.edu.cn.

INCLUDE WHEN CITING

Published online August 14, 2020; DOI: 10.3171/2020.6.JNS201957.

\section{Response}

No response was received from the authors of the original article.

CAANS 2021, except where prohibited by US copyright law

\section{Invasive neuromonitoring for poor-grade SAH}

TO THE EDITOR: We read with interest the article by Veldeman et al. ${ }^{1}$ (Veldeman M, Albanna W, Weiss M, et al. Invasive neuromonitoring with an extended definition of delayed cerebral ischemia is associated with improved outcome after poor-grade subarachnoid hemorrhage. J Neurosurg. Published online May 15, 2020. doi: 10.3171/2020.3.JNS20375). The authors conducted a comparative analysis of consecutive patients with poorgrade subarachnoid hemorrhage (SAH) allocated in two different cohorts, one before the implementation of invasive neuromonitoring (INM) and another after its introduction. The authors assessed the impact of INM on the rate of delayed cerebral ischemia (DCI) and on functional outcomes in these cohorts. The post-INM cohort suffered fewer DCI-related and silent infarctions, experienced better functional outcomes, and required fewer CT scans. However, the overall rate of DCI and mortality remained unchanged, whereas a nonsignificant upward trend in the incidences of CSF infections, number of endovascular rescue treatments (ERTs), and length of stay at the intensive care unit were observed.

Recently, we reported our experience in the use of cerebral microdialysis (CMD) in patients with poor-grade
SAH for the detection of ischemic crises leading to DCI. ${ }^{2}$ We advocated for a bilateral CMD policy and found that $24 \%$ of ischemic crises occurred in presumed silent hemispheres with few or no changes in the contralateral hemisphere. In addition, establishing a threshold of 7 or more ischemic events along the INM period yielded a specificity and sensitivity for DCI prediction of $96.2 \%$ and $83.3 \%$, respectively. According to previous reports and to the results of Veldeman et al., we noted that deleterious events were detected earlier and that fewer neuroimaging studies were needed..$^{3,4}$

The article by Veldeman et al. is definitely a valuable work that provides new insights and expands the potential applications of INM, while extending the classic definition of DCI. However, we would like to note some worthy concerns. First, it might be questionable to include patients who can be clinically assessed in a study focused on INM. Indeed, more than $60 \%$ of patients in the post-INM cohort were classified as Hunt and Hess grade 3. Second, it has not been disclosed which INM alteration was the basis for first-tier treatment to prevent DCI. Third, CMD reporting guidelines recommend presenting lactate/pyruvate ratio together with glucose as a first-tier approach to thoroughly characterize metabolic derangements. ${ }^{5}$ Fourth, although acknowledged by the authors, the rate of CSF infections is rather high in the post-INM cohort and was nearly double the acceptable infection rates for external ventricular drains. ${ }^{6}$ INM should be safe and reliable, therefore after the implementation of a novel INM policy, we would recommend a safety protocol to detect potential problems and establish proper solutions. In microbiological cultures of our 36 implanted CMD probes we did not find any infection. ${ }^{2}$ Lastly, the reasons leading to some of the clinical benefits obtained with the implementation of the INM are not thoroughly explained. For instance, why did the rate of silent infarctions significantly decrease? Was it due to an overall increase of ERT? Were patients requiring ERT comparable? As discussed by the authors, the main drawback of INM is that information refers to a limited area, whereas therapies are global. In that spirit, our team postulates that, regarding $\mathrm{CMD}$, there is a rationale for bilateral INM in patients with poor-grade SAH. ${ }^{2}$

Sergio García-García, MD ${ }^{1,2}$
Diego Culebras, MD
Ramón Torné, PhD'

\section{References}

1. Veldeman M, Albanna W, Weiss M, et al. Invasive neuromonitoring with an extended definition of delayed cerebral ischemia is associated with improved outcome after poorgrade subarachnoid hemorrhage. J Neurosurg. Published online May 15, 2020. doi:10.3171/2020.3.JNS20375

2. Torne R, Culebras D, Sanchez-Etayo G, et al. Double hemispheric microdialysis study in poor-grade $\mathrm{SAH}$ patients. Sci Rep. 2020;10(1):7466.

3. Helbok R, Kofler M, Schiefecker AJ, et al. Clinical use of cerebral microdialysis in patients with aneurysmal subarachnoid hemorrhage-state of the art. Front Neurol. 2017;8:565. 
4. Patet C, Quintard H, Zerlauth JB, et al. Bedside cerebral microdialysis monitoring of delayed cerebral hypoperfusion in comatose patients with poor grade aneurysmal subarachnoid haemorrhage. J Neurol Neurosurg Psychiatry. 2017;88(4):332-338.

5. Hutchinson PJ, Jalloh I, Helmy A, et al. Consensus statement from the 2014 International Microdialysis Forum. Intensive Care Med. 2015;41(9):1517-1528.

6. Kim JH, Desai NS, Ricci J, et al. Factors contributing to ventriculostomy infection. World Neurosurg. 2012;77(1):135-140.

\section{Disclosures}

The authors report no conflict of interest.

\section{Correspondence}

Sergio García-García: iskender_brave@hotmail.com.

\section{INCLUDE WHEN CITING}

Published online August 7, 2020; DOI: 10.3171/2020.5.JNS202047.

\section{Response}

We wish to thank Dr. García-García and colleagues for their interest in our research regarding INM and appreciate the critical appraisal of our recently published work. We have read their reply with great interest and would like to address the concerns raised by the authors.

As is highlighted by Dr. García-García and which is addressed in our paper, the Achilles heel of INM remains its low spatial resolution. Usually, to cover both anterior vascular territories, invasive probes are placed in the frontal watershed region ipsilateral to the ruptured aneurysm. The rationale behind this practice is based on the observation that the side of aneurysmal rupture, and resulting higher blood load, is most prone to DCI development. Midline aneurysms with a symmetrical blood load pose a problem in this regard. In a simulation of probe placement in 100 patients with SAH, Ulrich et al. concluded that probe location correlated with the territory of DCI ranging between 93\% for middle cerebral artery and $23 \%$ for basilar artery aneurysms. ${ }^{1}$ It is well appreciated that bilateral probe placement, by definition, increases the area under investigation, and we congratulate the authors on their recent important paper. However, the primary goal of this study was to establish for the first time whether the overall concept of INM in general is able to improve management and outcome in poor-grade $\mathrm{SAH}$. The benefit of multiple probes will have to be examined in future studies, taking into consideration the relative increase in risk in the context of additional probe placements and ideally the replacement with novel, noninvasive techniques.

Another safety issue is the risk of infection. We reported an increase of CSF infection of $10 \%(\mathrm{p}=0.098)$ after introduction of INM. In a previous trial at our institution, a rate of meningitis associated with external ventricular drains (EVDs) of 8.6 infections per 1000 drainage days was observed. ${ }^{2}$ The occurrence of meningoventriculitis has been significantly reduced by the use of chlorhexidine-containing dressing, similar to hygiene care for central lines, and this practice has been extrapolated to microdialysis probe care. ${ }^{3}$ However, within the context of the higher-grade SAH patient with extremely long hospitalization in the intensive care unit, CSF infection is mostly a diagnosis of exclusion, meaning that usually fever or elevated inflammatory markers trigger sampling, including culture of CSF. In cases of indwelling EVDs, the culturing of contaminants without pathological relevance is common. The nonsignificant increase in infections in our series could either be the result of coincidence or oversampling. However, we did not observe any overt infection of the probes either at the entry site or intraparenchymally. As the probes are not in direct contact with the ventricles, isolated ventriculitis in the absence of intraparenchymal infection implies that the infection rate, even if not artificially increased (see above), most likely developed irrespective of the probes. However, a contribution cannot be excluded. The brain tissue oxygenation $\left(\mathrm{ptiO}_{2}\right)$ monitoring system we use provides a bolt fixation mechanism completely immobilizing the probe, which is not the case for the microdialysis catheter. Small movements of the probe in and out of the skin remain possible, increasing the risk of infection by skin-derived pathogens. We have now introduced a multibolt fixation system for both monitoring systems (microdialysis and $\mathrm{ptiO}_{2}$ ) and will monitor the technical differences closely.

Hunt and Hess grade 3 hemorrhages represent a group of patients with a mild reduced state of consciousness. Apart from asymmetry in motor function, these patients cannot be thoroughly neurologically examined and their condition frequently deteriorates. Early placement of INM is considered for their high risk of deterioration and the need for therapeutic monitoring.

In daily practice we also recommend making treatment decisions based on multiple factors pointing in the same direction. The possibilities and number of molecules assessable in CMD are infinite, and current existing data underlines that the lactate-to-pyruvate ratio-due to its predictive value on outcome and infarct developmenthas been the parameter we focused on. For an elaborate metabolic assessment, glucose values are important; from a decision-making standpoint, management recommendations in the context of glucose are less homogenous. As noted by Cesarini et al., increased levels of glucose can also be associated with poor outcome ${ }^{4}$ However, the comment regarding the complexity of metabolic derangement, including the scenario of mitochondrial dysfunction, is well appreciated and will be the focus of additional work.

The concept of DCI and its complex pathophysiology is still only partially characterized. A growing body of data underlines that the phenomenon has to be seen as an affliction of the brain as a whole being predisposed to ischemic damage with superimposed local triggers. This uncertainty is reflected by the practice of first-tier systemic hypertension, but applying a localized endovascular treatment focused on resolving macroscopic vasospasm for refractory cases. The mere high-dosage delivery of nimodipine might exert protective effects independent of the macroscopic resolution of angiographic vasospasm. This is noted to emphasize that any diagnostic tool is only as valuable as the effectiveness of the treatment strategies it triggers. As the rate and modality of endovascular rescue treatment did not change from the historic to the present cohort, we believe that the decrease in the rate of previously silent 
infarctions can mostly be attributed to the higher vigilance and earlier treatment of deteriorating patients.

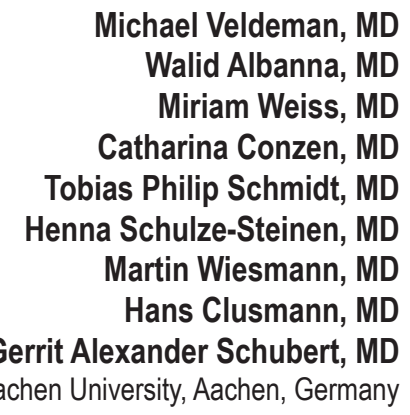

\section{References}

1. Ulrich CT, Fung C, Vatter H, et al. Occurrence of vasospasm and infarction in relation to a focal monitoring sensor in patients after SAH: placing a bet when placing a probe? PLoS One. 2013;8(5):e62754.

2. Scheithauer S, Bürgel U, Ryang YM, et al. Prospective surveillance of drain associated meningitis/ventriculitis in a neurosurgery and neurological intensive care unit. J Neurol Neurosurg Psychiatry. 2009;80(12):1381-1385.

3. Scheithauer S, Schulze-Steinen H, Höllig A, et al. Significant reduction of external ventricular drainage-associated meningoventriculitis by chlorhexidine-containing dressings: a before-after trial. Clin Infect Dis. 2016;62(3):404-405.

4. Cesarini KG, Enblad P, Ronne-Engström E, et al. Early cerebral hyperglycolysis after subarachnoid haemorrhage correlates with favourable outcome. Acta Neurochir (Wien). 2002;144(11):1121-1131.

\section{Reporting continuous variables and statistical significance in a study of 30-day hospital readmissions after mechanical thrombectomy for acute ischemic stroke}

TO THE EDITOR: We read with great interest the article by Mouchtouris et al. ${ }^{1}$ (Mouchtouris N, Al Saiegh F, Valcarcel B, et al. Predictors of 30-day hospital readmission after mechanical thrombectomy for acute ischemic stroke. J Neurosurg. Published online May 1, 2020. doi:10.3171/2020.2.JNS193249). In this study, it was concluded that hypertension, length of hospital stay, and hemorrhagic conversion were important prognostic factors for readmission within 30 days in acute ischemic stroke patients treated with mechanical thrombectomy. However, the following issues should be addressed carefully.

First, it can be seen from the descriptions in their Tables 1 and 2 that continuous variables are represented by means and standard deviations (SDs) and that the differences between groups are compared by an unpaired t-test.
However, we doubt whether those continuous variables described in these tables conform to a normal distribution and whether it is appropriate to describe those continuous variables that do not conform to a normal distribution as means and SDs. Obviously, the SD of some variables is almost equal to the mean, sometimes even larger than the mean. For example, the mean hospital length of stay in the readmitted group was 15.6 days, while the SD was 29.3 days, indicating that the data may not conform to a normal distribution. In this case, it is not appropriate to use an unpaired t-test to compare the differences between groups. We suggest using the Mann-Whitney U-test for statistical analysis of this study.

Second, as described in their Table 1, the hospital length of stay in the readmitted group was significantly higher than that in the non-readmitted group $(15.6 \pm 29.3$ vs $9.1 \pm 7.5$ days). However, the p value described in their article suggests no statistical difference between the two groups. Based on the data provided in Table 1, the $\mathrm{p}$ value recalculated by Stata 11 software is $<0.001$, which is notably different from the authors' results. Is this a statistical mistake? We look forward to receiving a reasonable explanation from the authors. In addition, since the followup time has been reported in this study, why not use Cox regression analysis ${ }^{2,3}$ to explore prognostic factors?

Siou Li, MD
Qing Xu, MD
Changhao Yin, MD
Mudanjiang Medical University, Affiliated Hongqi Hospital,
Heilongjiang, China
Heilongjiang Key Laboratory of Ischemic Stroke Prevention and
Treatment, Heilongjiang, China

\section{References}

1. Mouchtouris N, Al Saiegh F, Valcarcel B, et al. Predictors of 30-day hospital readmission after mechanical thrombectomy for acute ischemic stroke. J Neurosurg. Published online May 1, 2020. doi:10.3171/2020.2.JNS193249

2. Arts EE, Fransen J, Den Broeder AA, et al. Low disease activity (DAS28 $\leq 3.2$ ) reduces the risk of first cardiovascular event in rheumatoid arthritis: a time-dependent Cox regression analysis in a large cohort study. Ann Rheum Dis. 2017;76(10):1693-1699.

3. ElHafeez SA, Torino C, D'Arrigo G, et al. An overview on standard statistical methods for assessing exposure-outcome link in survival analysis (Part II): the Kaplan-Meier analysis and the Cox regression method. Aging Clin Exp Res. 2012;24(3):203-206.

\section{Disclosures}

The authors report no conflict of interest.

\section{Correspondence}

Changhao Yin: aoicg46@163.com.

INCLUDE WHEN CITING

Published online August 21, 2020; DOI: 10.3171/2020.5.JNS201857.

\section{Response}

We would like to thank $\mathrm{Li}$ et al. for their interest in our study and their critical review of our work, as well as 
the editor for enabling this scientific discourse. After thorough review of our data, we confirm that the continuous variables of age and National Institutes of Health Stroke Scale (NIHSS) scores are normally distributed, which was verified using histogram plots and the normal distribution curve. For that reason, the mean and SD are the appropriate descriptive statistics for these variables. We appreciate the authors' point regarding the hospital and ICU lengths of stay (LOS), both of which are not normally distributed, and the nonparametric Mann-Whitney U-test was performed to analyze them. The median value and interquartile range are reported for these variables in Table 1 in our article. Similarly, the median and interquartile range are the appropriate descriptive statistics for the time to readmission and readmission LOS (Table 2 in our article).

Furthermore, the hospital LOS was compared between patients who were readmitted and those who were not by using the Mann-Whitney U-test, and a statistically significant difference was seen with a p value of 0.045 (see Table 1). We appreciate the authors' suggestion of using the Mann-Whitney U-test; however, the suggested $\mathrm{p}$ value is not correct based on our data. However, this was updated and did not impact the rest of the statistical analysis.

Lastly, the Cox regression analysis was not performed because our study was not sufficiently powered to do so, and this was outside of our original scope. We aimed to identify significant risk factors of the binary outcome of readmission within 30 days from discharge. However, we certainly encourage larger-scale studies in the future that allow for such statistical analysis.

We thank Li et al. once again for their critical review, and we look forward to further research on this topic.

Nikolaos Mouchtouris, MD M. Reid Gooch, MD

Thomas Jefferson University and Jefferson Hospital for Neuroscience, Philadelphia, PA

INCLUDE WHEN CITING

Published online August 21, 2020; DOI: 10.3171/2020.7.JNS201917.

CAANS 2021, except where prohibited by US copyright law

\section{Intracranial pressure monitoring: challenge beyond the threshold numerical value}

TO THE EDITOR: We have read with great attention the article by Doron et al. ${ }^{1}$ (Doron O, Barnea O, Stocchetti N, et al. Cardiac-gated intracranial elastance in a swine model of raised intracranial pressure: a novel method to assess intracranial pressure-volume dynamics. J Neurosurg. Published online June 5, 2020. doi:10.3171/ 2020.3.JNS193262). We would like to congratulate the authors. Their paper is very relevant, and we agree with most of the authors' statements, especially with the description of a possible new model for monitoring intracranial pressure (ICP).
Our team believes that one great global need nowadays is to make ICP monitoring accessible to the largest number of patients possible for use in the treatment of many neurological and clinical diseases, not only neurological injury, because we believe that more widespread use of this method may ensure a better overall prognosis for these patients.

The article in question presents a study that uses small animals and a very small sample, and therefore the scientific evidence for using this ICP model clinically in human patients is still hypothetical. In addition, the method used by the authors of insufflation-deflation of the balloon is able to generate only one mechanism of a pathological process. The study did not define the borderline values of intracranial elastance that are associated with clinical findings. Details about the methodology used are also lacking, as it is still unknown if this method may be adversely affected by other parameters, in addition to the physiological ones already mentioned, like intracranial mass, hydrocephalus, and other clinical conditions.

Despite the search for a numerical limit value for ICP and new alternative models of monitoring, such as volume dynamics, by many in the scientific community, ${ }^{2,3}$ we believe that the ICP waveform (ICPwf) as a method of assessing and monitoring patients provides more accurate information than just the ICP cutoff number. The use of the ICPwf was associated with intracranial compliance (ICC), ${ }^{4,5}$ and in 2017 Ballestero et al. ${ }^{6}$ observed for the first time the relation between the peaks obtained with ICPwf monitoring, the $\mathrm{P} 2 / \mathrm{P} 1$ ratio, as a marker for studying hydrocephalus. This waveform can also be used in other cases, such as in patients with neoplasms, contusions, and intracranial trauma, in order to monitor ICC. Thus, the numerical values are more referential than conclusive, due to their variations and the limited information about the outcomes.

Technological advances in the medical field have allowed the creation of a new noninvasive model to monitor ICC, the B4C sensor (Braincare), which Ballestero et al. ${ }^{6}$ applied in their study. This model includes a tension meter and recorder connected to a mechanical device that touches the scalp surface in the frontoparietal area lateral to the sagittal suture. The ICP monitoring function of this instrument is based on the detection of brief changes in the measurements of the skull that result from pressure changes within it; that is, it relates the deformation of the skull with the detection of changes in mean ICP. ${ }^{6}$

We believe that this noninvasive ICPwf register technique would be of value to monitor neurocritical patients under various circumstances. Its advantages are affordability, efficiency, and simple and practical handling. Besides that, it does not trigger complications, such as infections or hemorrhages, which are frequent adverse effects from the use of invasive methods to monitoring ICP.

Nícollas Nunes Rabelo, MD Mateus Gonçalves de Sena Barbosa Atenas Medical School, Passos, MG, Brazil

Matheus Pereira Silva Lemos UNIFENAS Medical School, Alfenas, MG, Brazil 
Sérgio Brasil, MD, PhD Hospital das Clínicas, School of Medicine, University of São Paulo, Brazil

Gustavo Frigieri, MD, PhD Brain4Care, São Paulo, Brazil

\section{References}

1. Doron O, Barnea O, Stocchetti N, et al. Cardiac-gated intracranial elastance in a swine model of raised intracranial pressure: a novel method to assess intracranial pressure-volume dynamics. J Neurosurg. Published online June 5, 2020. doi:10.3171/2020.3.JNS193262.

2. Alperin NJ, Lee SH, Loth F, et al. MR-Intracranial pressure (ICP): a method to measure intracranial elastance and pressure noninvasively by means of MR imaging: baboon and human study. Radiology. 2000;217(3):877-885.

3. Kiehna EN, Huffmyer JL, Thiele RH, et al. Use of the intrathoracic pressure regulator to lower intracranial pressure in patients with altered intracranial elastance: a pilot study. $J$ Neurosurg. 2013;119(3):756-759.

4. Okon MD, Roberts CJ, Mahmoud AM, et al. Characteristics of the cerebrospinal fluid pressure waveform and craniospinal compliance in idiopathic intracranial hypertension subjects. Fluids Barriers CNS. 2018;15(1):21.

5. Westhout FD, Paré LS, Delfino RJ, Cramer SC. Slope of the intracranial pressure waveform after traumatic brain injury. Surg Neurol. 2008;70(1):70-74.

6. Ballestero MFM, Frigieri G, Cabella BCT, et al. Prediction of intracranial hypertension through noninvasive intracranial pressure waveform analysis in pediatric hydrocephalus. Childs Nerv Syst. 2017;33(9):1517-1524.

\section{Disclosures}

The authors report no conflict of interest.

\section{Correspondence}

Nícollas Nunes Rabelo: nicollasrabelo@hotmail.com.

\section{INCLUDE WHEN CITING}

Published online December 4, 2020; DOI: 10.3171/2020.9.JNS203395.

\section{Response}

We were pleased to read the comments of Nunes Rabelo and colleagues regarding our article and concur that the model provides a potentially useful platform to study intracranial pressure (ICP) dynamics. An advantage of the model we reported is that a large-animal swine model is used that has direct translational potential for patients with traumatic brain injury. As we discuss in the work, an inherent limitation of the swine model is that it usually precludes the use of large numbers of animals. As Nunes Rabelo and colleagues pointed out in their comments, our preliminary study did not allow for the definition of intracranial elastance thresholds to determine when the limits of intracranial volume reserve capacity have been reached. We agree wholeheartedly that this will be a crucial goal if the technology is to be applied in the clinical realm.

In their comments, Nunes Rabelo and colleagues stress the importance of ICP waveform analysis in helping to assess intracranial compliance. Although we agree with this point, it is important to point out that ICP waveform analysis alone has inherent limitations in its ability to provide a complete picture of intracranial compliance or its inverse, elastance. This is because the pressure change induced by the entry of blood volume into the cranium that the intracranial pulse pressure and waveform represent result from the effects of two parameters, the intracranial elastance and the blood volume entering the cranium. Without a direct measure of the intracranial elastance, the differing effects of these two parameters cannot be assessed in isolation. Hyperemia with high cerebral blood flow may lead to a higher intracranial pulse pressure while intracranial elastance remains unchanged.

Lastly, we concur with the assertion that an important goal is for ICP monitoring to be readily available to as many neurocritically ill patients worldwide as possible. We believe that it may be possible to incorporate the technology we describe into routine monitoring without using much in the way of additional resources. In fact, we are currently working to adapt the technology in a way that it can be used with any functioning external ventricular drain. Nunes Rabelo and colleagues advocate a noninvasive approach for monitoring ICP in order to potentially be able to spread monitoring availability to a wider range of patients. We support all efforts to develop noninvasive technologies for ICP monitoring, but at the same time, we are cognizant of the technological difficulties of achieving accurate measurements of ICP without a monitor within the cranium. We certainly hope that the approach advocated by Nunes Rabelo et al. will bear fruit and lead to important clinical advances. Our feeling is that these efforts should be pursued in parallel with efforts like ours that utilize a direct measure of intracranial elastance.

Guy Rosenthal, MD Omer Doron, MD Hadassah-Hebrew University Medical Center, Jerusalem, Israel

Nino Stocchetti, MD Milan University and Neuro ICU Fondazione IRCCS Cà Granda Ospedale Maggiore Policlinico, Milan, Italy

Ofer Barnea, PhD

Tel Aviv University, Tel Aviv, Israel

INCLUDE WHEN CITING

Published online December 4, 2020; DOI: 10.3171/2020.10.JNS203432.

CAANS 2021, except where prohibited by US copyright law

\section{The COVID-19 crisis and return to the Hippocratic Corpus}

TO THE EDITOR: The coronavirus disease 2019 (COVID-19) lockdown presented serious problems for neurosurgeons all over the world. I read with interest the article by Cenzato et al., ${ }^{1}$ which stressed the importance of the "hub-and-spoke system" (Cenzato M, DiMeco F, Fontanella M, et al. Editorial. Neurosurgery in the storm of COVID-19: suggestions from the Lombardy region, Italy (ex malo bonum). J Neurosurg. 2020;133[1]:33-34). In Lombardy this past February, hospitals were rapidly overcrowded by COVID-19 patients, and all neurosurgical activities (mainly trauma and vascular) were gathered at 
three centers (Niguarda, Brescia, and Varese), which were chosen based on geographical criteria as well as their receptive capacity, creating a hub-and-spoke scheme. In our hospital, scheduled craniotomies for patients with brain tumor were also postponed. I always experience fear and anxiety with regard to these patients' outcomes. In addition, one of our neurosurgeons was reassigned to the new COVID wards, adding to the new stress the anxiety and fear associated with potential infection.

One of the oldest neuroscientists may be Hippocrates (460-377 BC). The Hippocratic Corpus is a collection of the ancient Greek medical text attributed to Hippocrates. ${ }^{2}$ In this text, a typical case report of a man with phobia was described. "Nicanor's affection, when he went to a drinking party, was fear of the flute girl. Whenever he heard the voice of the flute begin to play at a symposium, masses of terrors rose up. He said that he could hardly bear it when it was night, but if he heard it in the daytime he was not affected. Such symptoms persisted over a long period of time." This phobia and anxiety were labeled as a medical disorder, which was clearly discriminated from major depression. ${ }^{2}$ Hippocrates taught his contemporaries how to achieve freedom from anxiety in his Hippocratic Corpus in which he mentions treatments including "stoicism," "peace of mind," and "freedom," which are the pillars of today's cognitive therapy. ${ }^{2}$ These three pillars would hold us all in good stead as we cope with the ramifications of the pandemic in our personal lives and professional practices. One way to escape from the clutches of anxiety is to devote one's attention to the present instead of worrying about the future. ${ }^{2}$

Even so, medical practitioners continue to be influenced by their clinical experience, not least in avoiding doing harm to patients. ${ }^{3}$ In these days of the COVID-19 pandemic, neurosurgeons must often face uncertainty in their practices when choosing which patients to prioritize for neurosurgical procedures. The advice given to us by Hippocrates even 25 centuries ago can be heeded to this day to enable us to cope with the impact of COVID-19 on the practice of neurosurgery.

Masanori Kurimoto, MD

Kurobe City Hospital, Toyama, Japan

\section{References}

1. Cenzato M, DiMeco F, Fontanella M, et al. Editorial. Neurosurgery in the storm of COVID-19: suggestions from the Lombardy region, Italy (ex malo bonum). J Neurosurg. 2020;133(1):33-34.

2. Crocq M-A. A history of anxiety: from Hippocrates to DSM. Dialogues Clin Neurosci. 2015;17(3):319-325.

3. Tsiompanou E, Marketos SG. Hippocrates: timeless still. $J R$ Soc Med. 2013;106(7):288-292.

\section{Disclosures}

The author reports no conflict of interest.

\section{Correspondence}

Masanori Kurimoto: m.kurimoto@med.kurobe.toyama.jp.

\section{INCLUDE WHEN CITING}

Published online January 8, 2021; DOI: 10.3171/2020.9.JNS203484.

\section{Response}

No response was received from the authors of the original article.

CAANS 2021, except where prohibited by US copyright law 